\title{
EXPLORING RICHTMYER-MESHKOV INSTABILITY PHENOMENA AND THE LINKS BETWEEN SURFACE PERTURBATIONS AND SHOCKED-INDUCED MASS EJECTION
}

\author{
SHI YINA, WANG PEI, ZHAO QIANG \& FENG QIJING \\ Institute of Applied Physics and Computational Mathematics, Beijing, P. R. China.
}

\begin{abstract}
This work investigates the mass ejected from surface perturbations as the shockwave reaches the AL-vacuum interface, which originates from unstable Richtmyer-Meshkov (RMI) impulse phenomena. The main purpose is to explore the relationships between the shockwave impulse and the geometric properties of surface perturbations, and how those relationships drive the total ejected mass, directionality and velocity distribution. We discuss in detail different types of surface geometry (sinusoidal, square-wave, chevron and semicircle), as well as the wavelengths and amplitudes of surface perturbation. The time evolutions of micro-jet ejection are simulated using a hydrodynamic Lagrangian-Remapping Eulerian method. The calculated results show that primary jetting ejection can be formed from the different shapes, and with increasing wavelength, the ejection mass keeps an increase while the jet head-velocity decreases. However, not all periodic perturbations behave similarly, and masses ejected from irregular surface cannot be normalized to its cross-sectional areas. The square-wave surface may yield pronounced, velocity-enhanced secondary jetting, which is a result of collision of primary jets.

Keywords: L-R two-step Eulerian method, mass ejection, metal, Richtmyer-Meshkov instability, surface perturbation
\end{abstract}

\section{INTRODUCTION}

Metals under shock-loaded conditions can lead to complex phenomena depending on the properties of material and shock conditions. We know that in one-dimensional (1D) geometry, the reflection of a planar shock front from a parallel, perfectly planar free surface accelerates this surface to approximately twice the particle velocity induced by the incident shock. In practice, material surface characteristics such as pits, bumps, voids, grooves, or scratches disrupt this 1D ideal case. When shock pressures are typically on the order of a few hundred thousand atmospheres, but gas pressures are about an atmosphere, the metal will release to a very low pressure at the surface as the shockwave reflects back into the metal whether there is a vacuum or a gas across the interface. Of interest is what happens to metals when a shock wave encounters different perturbed metal/vacuum interfaces. The phenomenon, which rapid ejection of micron-scale fragments usually happens from material surface, has attracted much attention since being discovered by Asay [1,2]. These ejected high-speed particles often have negative effects on optical and electrical measurements at metal surfaces, and are relevant to inertial confinement fusion.

This process of mass ejection has been extensively studied under explosive and impact loading. Previous research to understand ejecta sources has in most cases focused on experiment characterization and originated from a Richtmyer-Meshkov instability (RMI). The microjetting observed behind those perturbed surfaces may become one kind of main ejection modes. Experimental techniques include piezoelectric pins, optical shadowgraph, X-ray radiography and velocity measurements, et al. Some significant efforts have been dedicated to studying on the microjetting mechanism. The effects of groove shape, shock wave rise-time 
and shock pressure on micro-jet are investigated, including continuum level and molecular dynamics simulations [3, 4]. Recently, some measured results on ejecta particle size distributions are given [5], which are also predicted by percolation theory and MD simulations. Models aiming at a prediction of ejection velocity and mass are from early, empirical descriptions to modern theories based on the nonlinear RMI [6-9]. It is shown that the mass, size, and velocity of the ejecta vary depending closely on the surface status, initial shock conditions and the material properties.

In this work, we investigate the mass ejected from surface perturbations as the shockwave reaches the AL-vacuum interface. We discuss in detail the underlying mechanisms of surface microjetting related to different types of surface geometry (sinusoidal, square-wave, chevron groove and semicircle) as well as the wavelengths and amplitudes of surface perturbation. The time evolutions of micro-jet ejection are simulated using a hydrodynamic LagrangianRemapping Eulerian method. The effects of shockwave impulse as well as surface geometric properties on total ejected mass, directionality and velocity distribution are analyzed.

\section{MECHANISM AND MODELING OF MASS EJECTION}

\subsection{Methodology and modeling}

The simulation sample is metal aluminium with surface perturbation of initial wavelength $\lambda$ and amplitude $h$. Shock waves are generated by assigning the flyer plate an initial velocity to impact the target. The length of the flyer plate is much longer than that of the target to delay the effects of release fan originated from the free surface of the flyer plate. Here, the impact velocity is $3.4 \mathrm{~km} / \mathrm{s}$, and the impact pressure of aluminum samples is about $30 \mathrm{GPa}$. The initial configurations of our simulations are illustrated in Fig. 1. A proper propagation distance is set between the flyer plate and the bottom of periodic perturbation to ensure the formation of a supported shock wave before it encounters the tip.

The elastic-plastic hydrodynamic Lagrangian-Remapping two-step Euler method is used for the simulation of aluminium micro-jet ejection. The hydrocode uses dimensional split algorithm with Lagrangian re-map and is second order accurate in both time and space. A Young's or VOF approach is employed to capture the interface. The strengths of the code lie in its ability to capture large-scale material deformation, wave propagation and interaction generated from impact phenomena. For the aluminium material, the plastic stress-strain

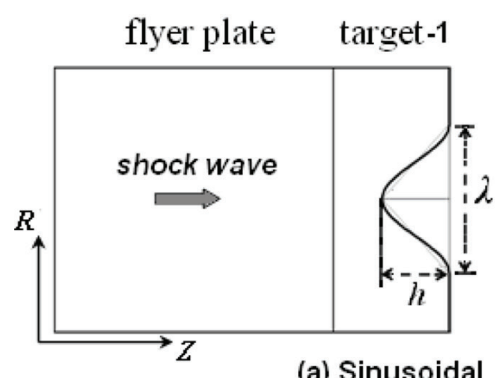

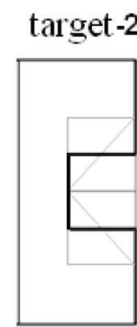

(b) Square

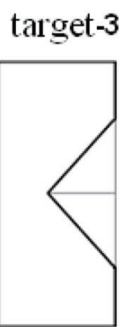

(c) Chevron target-4

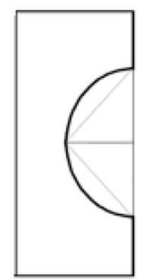

(d) Semicircle

Figure 1: The shapes of initial surface perturbation. (a) Sinusoidal; (b) Square-wave; (c) Chevron and (d) Semicircle. 
relation is modelled by the von Misses yield criterion, and the yield strength of the material $\sigma_{Y}$ is determined by the Johnson-Cook (JC) model, which can be expressed as

$$
\begin{gathered}
S_{r r}^{2}+S_{z z}^{2}+S_{\theta \theta}^{2}+2 S_{r z}^{2} \leq(2 / 3) \sigma_{Y}^{2} . \\
\sigma_{Y}=\left[A+B\left(\varepsilon^{p}\right)^{n}\right] \cdot\left[1+C \ln \left(\dot{\varepsilon}^{p} / \dot{\varepsilon}_{0}\right)\right] \cdot\left[1-\left(T^{*}\right)^{m}\right] .
\end{gathered}
$$

where parameters $A, B, C, m$ and $n$ are determined by experiments. $\varepsilon^{p}$ and $\dot{\varepsilon}^{p}$ are the equivalent plastic strain and strain-rate, $\dot{\varepsilon}_{0}=1.0 \mathrm{~s}^{-1}, T^{*}=\left(T-T_{\mathrm{r}}\right) /\left(T_{\mathrm{m}}-T_{\mathrm{r}}\right), T_{\mathrm{m}}$ is the melting temperature and $T_{\mathrm{r}}$ is the room temperature,. The equation of state (EOS) is adopted by the Mie-Gruneisen (MG) equation as

$$
\begin{gathered}
p=\frac{\rho_{0} c_{0}^{2} \mu\left[1+\left(1-\frac{\gamma_{0}}{2}\right) \mu-\frac{a}{2} \mu^{2}\right]}{\left[1-\left(S_{1}-1\right) \mu-S_{2} \frac{\mu^{2}}{\mu+1}-S_{3} \frac{\mu^{3}}{(\mu+1)^{2}}\right]^{2}}+\left(\gamma_{0}+a \mu\right) E, \rho \geq \rho_{0} . \\
p=\rho_{0} c^{2} \mu+\left(\gamma_{0}+a \mu\right) E, \quad \rho<\rho_{0} .
\end{gathered}
$$

where $\mu=\rho / \rho_{0}-1, \rho_{0}$ is the initial density, $c_{0}$ is the initial sound velocity, and $a, \gamma_{0}$ are the Gruneisen coefficients. $S_{1}, S_{2}$ and $S_{3}$ are the parameters that relate the shock speed to the particle velocity, and $E$ is the initial internal energy.

\subsection{Mechanisms of microjetting}

We first carry out the simulations of microjetting ejection from chevron perturbation surface of $h=2.0 \mu \mathrm{m}, \lambda=2.31 \mu \mathrm{m}$, to illustrate jetting mechanisms. The chevron groove is one kind of common surface profile obtained from a machining process. When a shock wave breaks out at a groove tip, a series of rarefaction waves propagate into the target interior. The matters near the groove free surface acquire $R$-component velocities and move toward the axis of symmetry, as shown in Fig. 2(a). Consequently, the matters above and below the axis collide

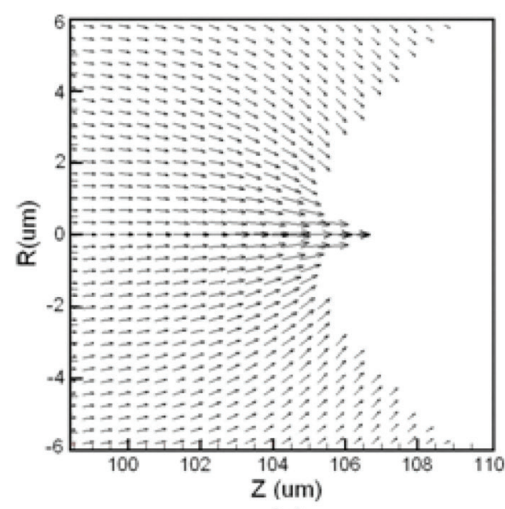

(a)

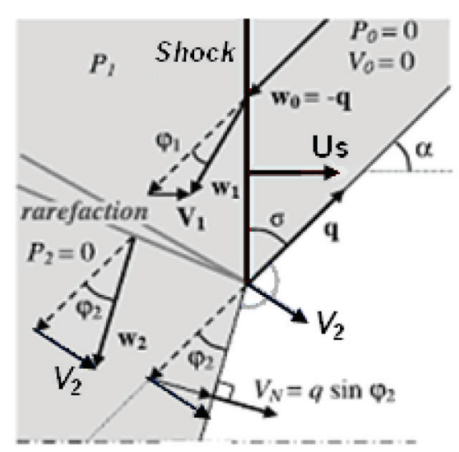

(b)

Figure 2: (a) Velocity vector plot near the tip of the chevron groove; (b) Two-dimensional analytical description in the steady-state regular case. 
around the axis, along with the central, fast, forward-moving matters, forming a high-speed jet along the shock direction.

The main features governing wave reflection from the groove surface can be approached by shock wave theory. Figure 2(b) is the two-dimensional description of the interaction of a planar shock wave (shock pressure $P_{1}$, shock velocity $U_{\text {s }}$, particle velocity $V_{1}$ ) with a planar free surface at an incidence angle $\sigma$. In the regular case, the shocked state is below the sonic point, and the flow is supersonic, which allows steady-state conditions where velocities and angles remain constant during the interaction. By neglecting pressure decay during the interaction of the planar shock with the groove edge, the resulting jet velocity can be estimated. In Fig. 2(b), $q=U_{\mathrm{s}} / \sin \sigma$ is the shock apparent velocity along the interface, $\alpha=90^{\circ}-\sigma$ is the groove half-angle, $\varphi_{2}$ is the deviation angle after full release and $V_{2}$ is the collapse velocity along the bisecting direction, which can be derived from its normal component.

A spatial distribution of the jet velocity $\left(V_{\mathrm{R}}\right.$ or $\left.u_{\mathrm{z}}\right)$ is plotted in Fig. 3(b), showing acceleration near the groove, reduced acceleration further away, and deceleration within the jet head. The acceleration is close to linear, and $u_{\mathrm{z}}$ is symmetric about the axis of symmetry, while $V_{\mathrm{R}}$ shows negative gradients along the z-axis near the groove tip [Fig. 3(a)].

\section{RESULTS AND DISCUSSION}

\subsection{Surface geometry effects on mass ejection}

With the above model and methods, we conducted a variety of numerical simulations to explore the underlying mechanisms of surface microjetting related to different types of surface geometry, including sinusoidal, square-wave, chevron and semicircle. Figure 4 is simulation results of the micro-jet morphology evolution from different perturbation surface of $h=2.0 \mathrm{~mm}, \lambda=4.0 \mathrm{~mm}$. It shows that primary jetting ejection can be formed from these different shapes, and the growth in lateral and longitudinal dimensions followed by necking. The former is accompanied by pronounced mass accumulation, and then, the necking is the dominant phenomenon. Both mass accumulation and necking are due to the velocity gradi-

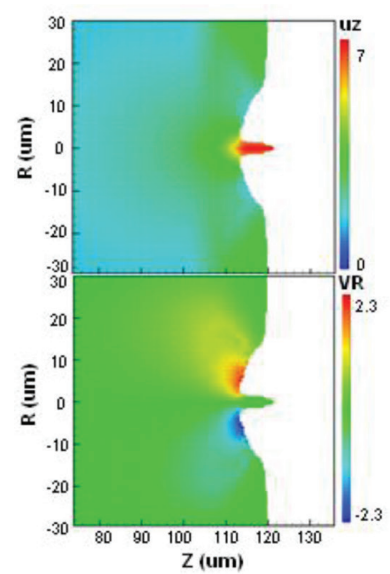

(a) (b)

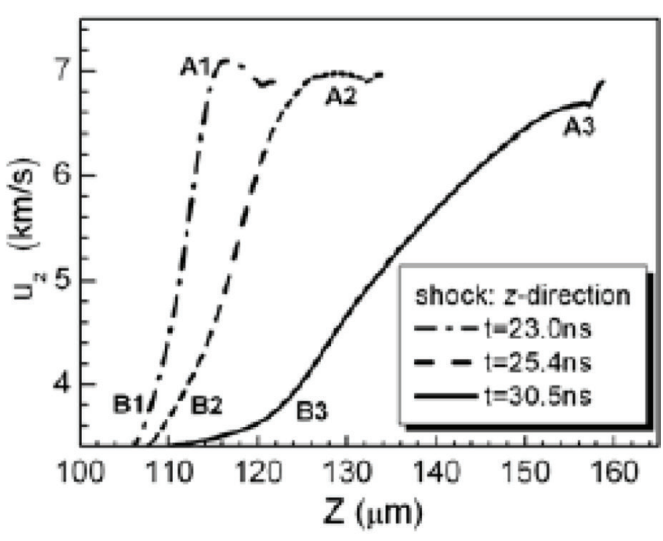

Figure 3: (a) 2D maps of microjetting velocity (in $\mathrm{km} / \mathrm{s}$ ) at $t=20 \mathrm{~ns}$; (b) Spatial distribution of jet velocity along the $z$-direction at different time. 
ents along jet stretching direction, as seen in Fig. 3(b): the gradient values in jet different region give rise to two competing phenomena: mass accumulation in the head (A1, A2 and A3) and necking (B1, B2 and B3) behind it. The decrease in their amplitudes as increasing time leads to reduced mass accumulation and increased necking at later times. Especially, for the square-wave perturbation, two jetting stages can be clearly identified: in the first stage seen as Fig. 4(b), two jets form at the left and right corners within a short period, and the jetting becomes relatively stable; in the second stage, the first two jets collide, leading to a stable, velocity-enhanced, secondary jet. The jet head velocity evolutions are compared in Fig. 5. Secondary jet from square-wave perturbation surfaces can greatly enhance jetting velocity ratio.

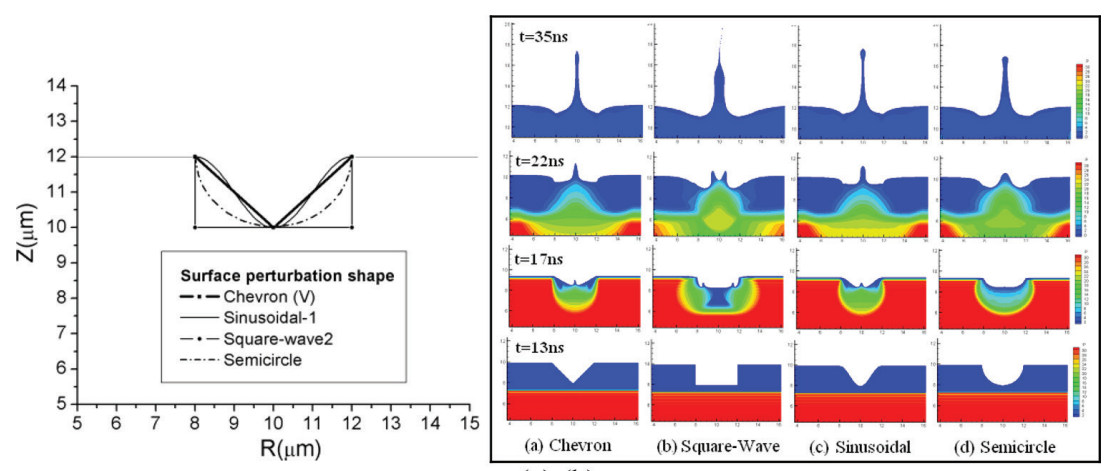

(a) (b)

Figure 4: Jet evolution for different surface shapes with $h=2.0 \mathrm{um}, \lambda=4.0 \mu \mathrm{m}$. (a) Surface perturbation shape; (b) Simulation results at $t=35 \mathrm{~ns}$.

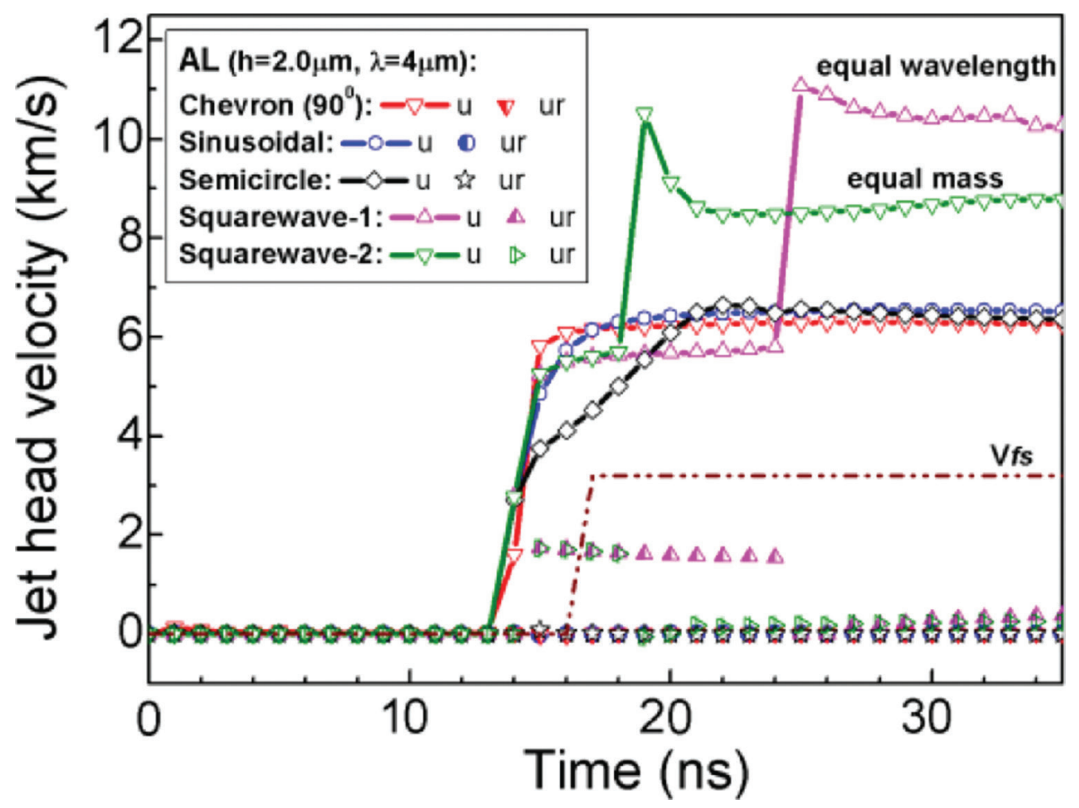

Figure 5: Effect of initial perturbation shapes on jet head velocity evolution. 


\subsection{Perturbation wavelength and amplitude dependences of mass ejection}

Here, we also carried out some simulations to investigate the effects of perturbation wavelengths and amplitudes on mass ejection. We consider the four cases of different perturbation wavelength and amplitude with the same impact velocity. The ratios of amplitude and wavelength are $0.25,0.5$ and 1.0, respectively. Figure 6 is simulation results of the micro-jet morphology evolution under the above perturbation parameter conditions. The calculated results are compared between the standard sinusoidal and square-wave surface.

The jet head velocity evolutions and mass accumulation are shown in Fig. 6. It can be seen that for the sinusoidal surface, with increasing wavelength, the maximum and asymptotic values of jet velocity $(u, u z)$ decrease, while the time durations to reach the asymptotic or stable velocity increase. The reason is that, at smaller wavelengths, $V_{\mathrm{R}}$ is larger so the jet head gains more acceleration and reaches the peak velocity within a shorter amount of time. For the chevron and semicircle shapes, there are the similar jetting features. However, the jet head velocity evolution is very different from the square-wave surface in the early stage and later secondary jetting stages. Figure 7 compares the jet mass accumulation for the four different wavelength and amplitude ratios. The results indicate that with increasing wavelength, the ejection mass keeps an increase while the jet head-velocity decreases. The square-wave
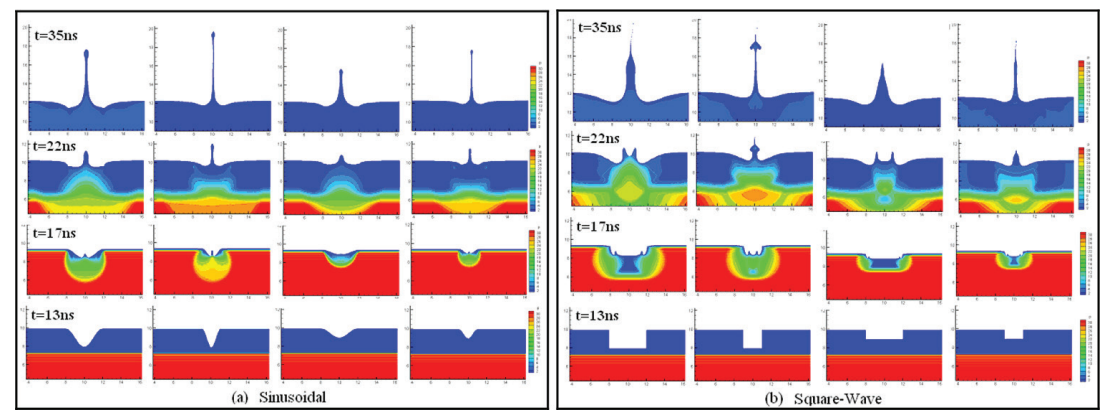

Figure 6: Microjetting evolutions for different ratio of perturbation amplitude and wavelength. (a) Sinusoidal shape; (b) Square-wave shape.
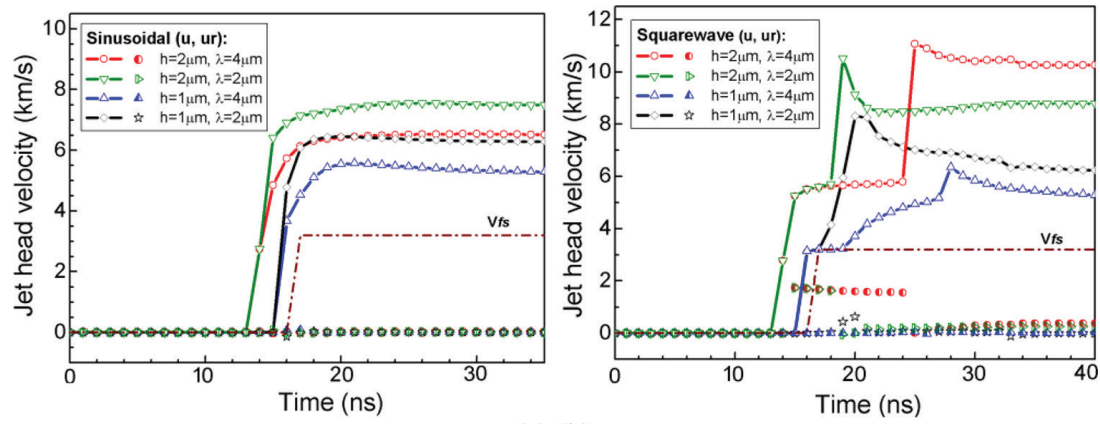

(a) (b)

Figure 7: Effect of initial surface perturbation parameters on jet head velocity evolutions. (a) Sinusoidal shape; (b) Square-wave shape. 

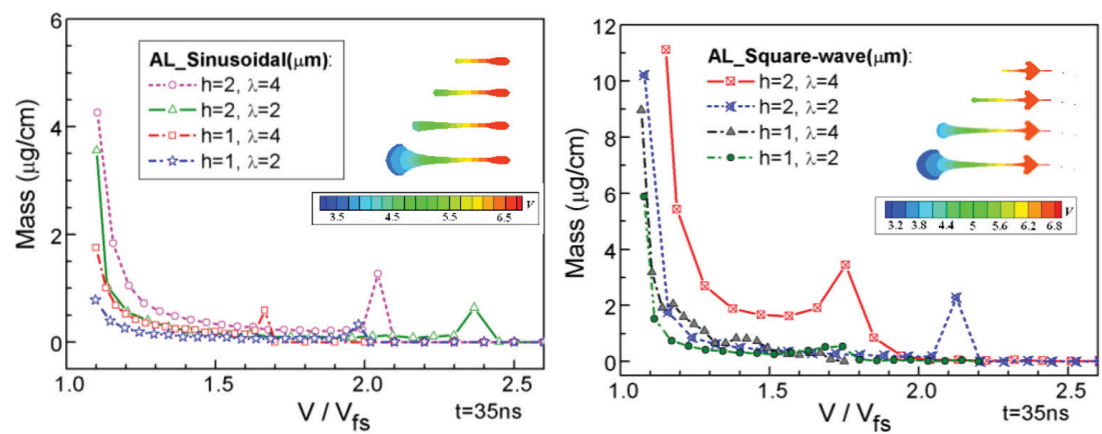

(a) (b)

Figure 8: Ejection mass vs. velocity for different ratio of perturbation amplitude and wavelength. (a) Sinusoidal shape; (b) Square-wave shape.

surface may yield pronounced, velocity-enhanced secondary jetting, and the total ejecta mass increases more significantly, which is a result of collision of primary jets.

\section{CONCLUSION}

This work investigates the mass ejected from surface perturbations as the shockwave reaches the AL-vacuum interface, which originates from unstable Richtmyer-Meshkov impulse phenomena. Using a hydrodynamic L-R two-step Eulerian method, we explore in detail the underlying mechanisms of surface microjetting related to different types of surface geometry (sinusoidal, square-wave, chevron groove and semicircle) as well as the wavelengths and amplitudes of surface perturbation. The calculated results show that primary jetting ejection can be formed from different perturbation surfaces, and with increasing wavelength, the ejection mass keeps an increase while the jet head-velocity decreases. The fundamental mechanism for jet formation is that transverse collision induces high stress concentrations, and the resulting strong stress gradient near the metal/vacuum interface. However, not all periodic perturbations behave similarly, and masses ejected from irregular surface cannot be normalized to its cross-sectional areas. For the square-wave surface, secondary jetting ejection can be clearly identified: in the first stage, relatively stable primary jets; in the second stage, the first two jets collide, leading to a stable, velocity-enhanced, secondary jet. The total ejecta mass increases more significantly, which is a result of collision of primary jets.

\section{ACKNOWLEDGEMENTS}

This work was supported by the National Natural Science Foundation of China (No.11371067) and the Science and Technology Development Foundation of China Academy of Engineering Physics (No.2015B0201044).

\section{REFERENCES}

[1] Walsh, J.M., Shreffler, R.G. \& Willig, F.J., Limiting conditions for jet formation in high velocity collisions. Journal of Applied Physics, 124(3), pp. 349-359, 1953. https://doi.org/10.1063/1.1721278

[2] Asay, J.R., Material ejection from shock-loaded free surfaces of aluminum and lead. Report No. SAND-76-0542, 1976.

https://doi.org/10.2172/7136578 
[3] Dyachkov, S.A., Parshikov. A.N. \& Zhakhovsky, V.V., Shock-produced ejecta from tin: comparative study by molecular dynamics and smoothed particle hydrodynamics methods. Journal of Physics: Conference Series, 653, pp. 012043, 2015. https://doi.org/10.1088/1742-6596/653/1/012043

[4] Durand, O. \& Soulard, L., Large-scale molecular dynamics study of jet breakup and ejecta production from shock-loaded copper with a hybrid method. Journal of Applied Physics, 111, pp. 044901, 2012. https://doi.org/10.1063/1.3684978

[5] Sorenson, D.S., Minich, R.W., Romero, J.L., Tunnell, T.W. \& Malone, R.M., Ejecta particle size distributions for shock loaded Sn and AL Metals. Journal of Applied Physics, 92(10), pp. 5830-5836, 2002. https://doi.org/10.1063/1.1515125

[6] Han, C.S., A semi-empirical equation for estimating the micro-jet ejection from shocked free surface. Chinese Journal of High Pressure Physics, 3(3), pp. 234-240, 1989.

[7] Buttler, W.T., Oro, D.M., Preston, D.L., Mikaelian, K.O., Cherne, F.J., Hixson, R.S., Mariam, F.G., Morris, C., Stone, J.B., Terrones, G. \& Tupa, D., Unstable RichtmyerMeshkov growth of solid and liquid metals in vacuum. Journal of Fluid Mechanics, 703, pp. 60-84, 2012.

[8] Shi, Y.N., Qin, C. S., Liu, C. \& Feng, Q.J., Study on fragment size of micro-jet from shocked metal surface with groove defect. Acta Armamentar II, 34, pp. 280-286, 2013.

[9] Dimonte, G., Terrones, G., Cherne, F.J. \& Ramaprabhu, P., Ejecta source model based on the nonlinear Richtmyer-Meshkov instability. Journal of Applied Physics, 113, pp. 024905, 2013.

https://doi.org/10.1063/1.4773575 